\title{
Current developments and future directions in COPD
}

\author{
Alexander G. Mathioudakis (10 ${ }^{1,2}$, Lowie E.G.W. Vanfleteren $\mathbb{1}^{3}$, Lies Lahousse $\mathbb{1}^{4}$, \\ Andrew Higham (10) ${ }^{1,2}$, James P. Allinson ${ }^{5}$, Carolina Gotera ${ }^{6}$, Dina Visca (1) ${ }^{7,8}$, \\ Dave Singh ${ }^{1,2,9}$ and Antonio Spanevello 7,8
}

\begin{abstract}
Affiliations: 'Division of Infection, Immunity and Respiratory Medicine, School of Biological Sciences, The University of Manchester, Manchester, UK. ${ }^{2}$ North West Lung Centre, Wythenshawe Hospital, Manchester University NHS Foundation Trust, Manchester Academic Health Science Centre, Manchester, UK. ${ }^{3}$ COPD Center, Institute of Medicine, Sahlgrenska University Hospital, University of Gothenburg, Gothenburg, Sweden. ${ }^{4}$ Dept of Bioanalysis, Pharmaceutical Care Unit, Ghent University, Ghent, Belgium. ${ }^{5}$ The Royal Brompton Hospital and The National Heart and Lung Institute, Imperial College London, London, UK. ${ }^{6}$ Dept of Pneumology, IIS-Fundación Jiménez Díaz, ISCIII-CIBERES, Madrid, Spain. ${ }^{7}$ Division of Pulmonary Rehabilitation, Istituti Clinici Scientifici Maugeri, IRCCS, Tradate, Italy. ${ }^{8}$ Dept of Medicine and Surgery, Respiratory Diseases, University of Insubria, Varese-Como, Italy. ${ }^{9}$ Medicines Evaluation Unit, Manchester, UK.
\end{abstract}

Correspondence: Alexander G. Mathioudakis, North West Lung Centre, Wythenshawe Hospital, Manchester University NHS Foundation Trust, Southmoor Road, Manchester, M23 9LT, UK.

E-mail: alexander.mathioudakisaManchester.ac.uk

@ERSpublications

Current developments and future directions in COPD: a critical summary of some of the most recent ground-breaking research studies and policy documents from @ERSpublications

https://bit.ly/3oW0xDM

Cite this article as: Mathioudakis AG, Vanfleteren LEGW, Lahousse L, et al. Current developments and future directions in COPD. Eur Respir Rev 2020; 29: 200289 [https://doi.org/10.1183/16000617.0289-2020].

ABSTRACT The European Respiratory Society journals publish respiratory research and policy documents of the highest quality, offering a platform for the exchange and promotion of scientific knowledge. In this article, focusing on COPD, the third leading cause of death globally, we summarise novel research highlights focusing on the disease's underlying mechanisms, epidemiology and management, with the aim to inform and inspire respiratory clinicians and researchers.

\section{Introduction}

COPD, the third leading cause of death globally, is associated with a significant burden to patients and society $[1,2]$. The development of novel treatments is hindered by the complexity and heterogeneity of the disease [3, 4]. COPD is the subject of extensive research, aiming to improve our understanding of underlying disease mechanisms and to identify effective, targeted treatments. For a respiratory clinician or researcher, it is challenging to keep up to date with the research advances being made in this field. In this review, we critically summarise some of the most recent, high-quality research studies and policy documents published in the European Respiratory Society (ERS) journals, aiming to inform and inspire clinicians and researchers.

This article was produced in collaboration with the ERS Early Career Members Committee, and follows and complements the cutting edge research presented from the ERS International Congress that was published in the ERJ Open Research [5-14].

Provenance: Commissioned article, peer-reviewed

Received: 1 Sept 2020 | Accepted after revision: 27 Oct 2020

Copyright $\odot$ ERS 2020. This article is open access and distributed under the terms of the Creative Commons Attribution Non-Commercial Licence 4.0. 


\section{Epidemiology}

The global prevalence of COPD is increasing with an estimated mean prevalence of $13 \%$ among the general population aged $\geqslant 40$ years $[2,15,16]$. By interpolating data from the Geographic Information System, BLANCO et al. [15] were able to visualise differences in COPD prevalence by continent ranging from $12 \%$ in Europe to $14 \%$ in Africa. Population growth, global ageing and cumulative exposure to risk factors may explain this increasing COPD prevalence worldwide [17]. COPD risk factors may change over time with new forms of smoking and vaping, and by location with differences in air pollution by region. The contribution of outdoor air pollution on COPD prevalence was reported by a recent UK Biobank analysis [18]. Among 303887 individuals included, aged 40-69 years, higher exposure to pollutants was significantly associated with higher COPD prevalence. Interestingly, higher ambient concentrations of particulate matter and nitrogen dioxide had a larger impact in obese, lower income and non-asthmatic participants [18]. Synergistic effects between excess body fat and pollutants might indeed enhance inflammatory responses causing more airway damage [18]. Furthermore, participants with a lower income might be particularly prone to COPD due to poor nutrition, more respiratory infections during childhood, worse housing conditions, or more occupational exposures [18]. Regarding occupational exposures, another interesting analysis of the UK Biobank data observed an increase in COPD risk for jobs with a high potential of exposure to noxious vapours and (diesel exhaust) fumes, dusts, pesticides and herbicides including painters, plastic moulders, warehouse stackers, food processors, farmers and gardeners [19]. Besides outdoor pollution and occupational exposure, indoor air pollution remains a major COPD risk factor, especially in low- and middle-income countries. An observational study in rural Kyrgyzstan in Central Asia, observed that COPD was more than three times as prevalent among highlanders $(\sim 2050 \mathrm{~m}$ above sea level) compared to lowlanders ( $750 \mathrm{~m}$ above sea level, $36.7 \%$ versus $10.4 \%$ respectively; $\mathrm{p}<0.001$ ) [20]. Since high altitude might require more (solid fuel-based) heating and limit ventilation possibilities, they observed a higher amount of indoor particulate matter particles with a 50\% cut-off aerodynamic diameter of $2.5 \mu \mathrm{m}\left(\mathrm{PM}_{2.5}\right)$ in highland compared to lowland houses [20]. This household biomass exposure might affect women more, in line with a higher percentage of females among COPD participants in highlands versus lowlands (51\% versus 20\%) [20]. Within the UK Biobank analysis, the association between $\mathrm{PM}_{2.5}$ and COPD for females was stronger leaving the possibility that women are both more frequently exposed and more vulnerable towards higher concentrations of fine particulate matter. Besides differences in smoking habits, these differences in exposure to air pollutants require further investigation to understand COPD phenotypes in females. Women often present with pronounced dyspnoea and comorbidities such as anxiety, depression or osteoporosis [21]. In a pulmonary rehabilitation programme, females had a higher number of comorbidities than male COPD patients [22]. COPD under-diagnosis is more frequent in females than males and this requires increased awareness. Analysis of global internet searches using Google Trends demonstrated a lack of awareness of COPD in the general population compared to diabetes for example [23]. This awareness is crucial to promote prevention by smoking cessation, to enable early diagnosis and to tailor treatment accordingly. COPD mortality trends are decreasing in nearly all countries [24]. However, you can only die from COPD if you have been diagnosed with COPD (by spirometry) during life. We also need to be aware that if by 2040 more people die "with" rather than "from" COPD, comorbidities aggravated by COPD deserve increased attention to reduce all-cause early mortality in patients with COPD [24].

COPD is strongly associated with cardiovascular disease including acute myocardial infarction (AMI), heart failure and arrhythmias [25]. Relative to individuals with normal lung function, COPD patients with Global Initiative for Chronic Obstructive Lung Disease (GOLD) stage 2-4 had increased all-cause mortality (HR 1.7, 95\% CI 1.4-2.1) and cardiovascular mortality (HR 2.1, 95\% CI 1.2-3.6) [26]. Prognosis of patients with COPD after AMI remains worse compared to patients without COPD and after correction for known prognostic factors and smoking [25]. Therefore, it remains important to develop clinically useful biomarkers to better identify COPD patients at higher risk of death. WASCHKI et al. [27] studied 2085 patients with stable COPD from the multicentre COSYCONET cohort study and found high-sensitivity troponin I (hs-TnI) to be a strong predictive marker for all-cause mortality after adjusting for airflow limitation, dyspnoea, exercise capacity, severe exacerbations and cardiovascular risk factors. Although the monitoring of clinical characteristics such as age, dyspnoea and airflow obstruction predicted 3-year all-cause mortality in COPD patients from the Health Improvement Network cohort [28], hs-TnI might predict very early pathology in myocytes not covered by other biomarkers [27]. We probably need to give up on seeking one-marker-which-serves-all for COPD patients [29]. For example, the serial assessment of the age, dyspnoea and airflow obstruction score seemed especially informative for COPD patients who smoke, were depressed or had lower body mass index, while measuring hs-TnI might be more informative in dyspnoeic COPD patients with frequent exacerbations, right ventricular dysfunction or hypoxaemia present $[27,28]$. Given the heterogeneity in COPD, it remains difficult to align severity with disease activity for all patients in the same manner and different mechanisms might drive individuals' 
prognostic risk. Using different disease biomarkers might enhance the delivery of individualised therapy for patients with COPD [29].

While the severity of airflow limitation is often used as clinical trial entry criterium, the assessment of comorbidities, the symptom burden and exacerbation history are also required when planning treatment [30]. To better assess comorbidities in COPD, we need to agree upon more standardised tests and clinical useful cut-off points which identify for example, the clinically important loss of muscle mass and function [31]. In addition, the presence of multiple diseases may be linked by common underlying mechanisms but we still limit the generation of evidence towards treating such multimorbidity because we both choose inadequate study outcomes [32] and exclude patients with these comorbidities from COPD randomised clinical trials (RCTs) [30]. To improve prognosis of COPD patients, we therefore need to be more inclusive and better understand the heterogeneity present in the overall COPD, being aware that some COPD patients are less well represented in analyses. A comparison of those with incomplete versus complete UK Biobank data demonstrated that data was more often missing for current smokers with lower education, lower income or with an occupation at risk of COPD [18].

\section{Basic mechanisms}

COPD is caused by the chronic inhalation of cigarette smoke or other harmful particles, which cause pulmonary injury, leading to chronic airway inflammation and tissue remodelling. The major histopathological changes observed include small airway disease and emphysema. Recent advances have shown that small airway disease precedes emphysema, suggesting a temporal pattern of disease progression $[33,34]$. The mechanisms which drive these changes are poorly understood. However, the role of microbial colonisation, frequent exacerbations and the type of inflammation present are emerging as important drivers of tissue inflammation and remodelling [35].

Inflammation is a dichotomous process; offering protection against pathogens whilst also increasing the potential for unwanted tissue injury (figure 1). Airway neutrophilia is associated with the severity of airflow limitation [36]. Activated neutrophils release proteases which cause tissue damage. Wooding et al. [37] reported that COPD blood neutrophils are more susceptible to activation. When exposed to diesel exhaust particles, the expression of neutrophil activation markers including CD16, CXCR2 and CD11b, were increased in blood neutrophils from COPD patients but not healthy controls. Regulating neutrophil

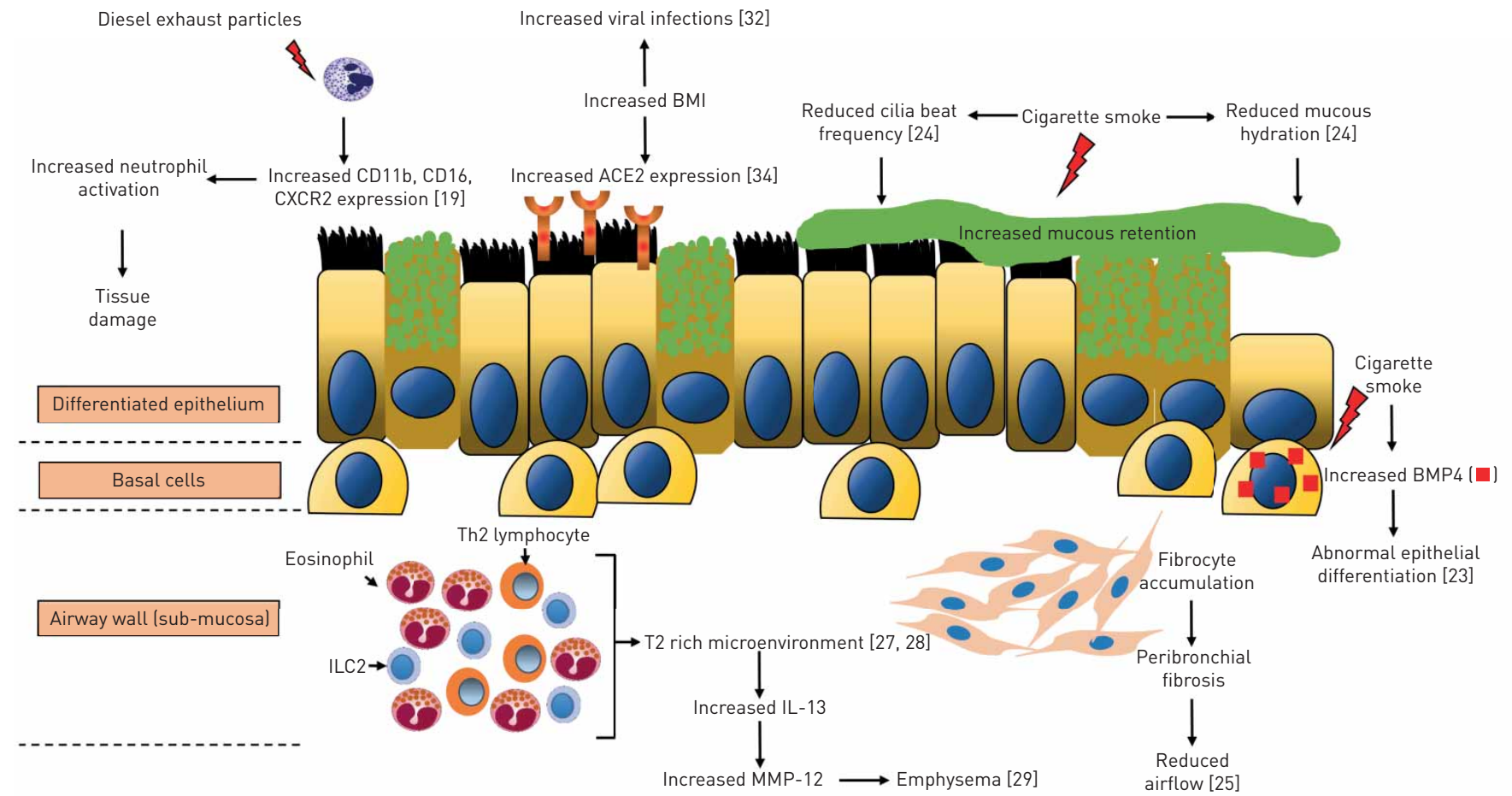

FIGURE 1 Basic mechanisms. Schematic diagram illustrating some of the studies published in European Respiratory Society journals which provide important insights into the basic mechanisms of COPD pathophysiology. ACE2: angiotensin converting enzyme 2; BMI: body mass index; BMP4: bone morphogenetic protein 4; IL-13: interleukin-13; ILC2: type 2 innate lymphoid cell; MMP-12: matrix metalloproteinase-12; T2: type 2; Th2: T-helper 2 lymphocytes. 
activity may be important to protect lungs from unwanted tissue damage during inflammation and club cell secretory protein may be important in this regard. KNABE et al. [38] reported that club cell secretory protein reduces neutrophil chemotaxis towards cigarette smoke-induced CXCL8 release from bronchial epithelial cells. Similarly, STARKEY et al. [39] demonstrated that cigarette smoke-exposed interleukin (IL)-22 deficient mice have reduced lung neutrophil numbers, and are protected from airway fibrosis and alveolar tissue destruction, suggesting a role for IL-22 in neutrophil infiltration. The authors also showed that patients with COPD have increased pulmonary IL-22 ligand and receptor expression compared to controls, suggesting a role for IL-22 in regulating pulmonary inflammation and tissue injury in COPD. However, IL-22 may also protect against microbial infection [40] and further research is required to understand the exact role in COPD.

Cigarette smoke exposure damages the airway epithelium. Repair of the airway epithelium requires re-epithelialisation with differentiated cells types, a process orchestrated by basal cells. Cigarette smoke alters the differentiation programme of basal cells, upregulating bone morphogenetic protein 4 which inhibits differentiation into normal ciliated and secretory cells [41]. This may explain why cigarette smoke reduces mucociliary transport in smoke-exposed bronchial epithelial cells in vitro, and in a ferret model of COPD [42]. The authors observed reduced cilia beat frequency, and reduced mucous hydration resulting in more viscous mucous which is more difficult to remove from the airways. This will result in increased airflow limitation and act as a reservoir for microbial colonisation.

Myofibroblasts mediate wound repair. During chronic inflammation, this process can cause increased collagen deposition in the extracellular matrix, causing tissue fibrosis. Myofibroblasts are derived from sub-epithelial fibroblasts, epithelial cells which undergo epithelial-mesenchymal transition, or circulating fibrocytes produced by the bone marrow. The number of sub-epithelial fibrocytes are increased in the proximal and distal airway walls of patients with COPD compared to controls [43]. In addition, the numbers of fibrocytes negatively correlate with lung function and positively correlate with airway wall thickness suggesting a role for these cells in peribronchiolar fibrosis. The extracellular matrix also contains large amounts of hyaluronic acid, a glycosaminoglycan. During COPD exacerbations the levels of circulating hyaluronic acid and hyaluronidase-1 (the enzyme which degrades hyaluronic acid) increase compared to the stable state [44]. This suggests that tissue inflammation during an exacerbation alters the extracellular matrix, leading to further airway remodelling.

Eosinophilic lung inflammation occurs in a sub-group of COPD patients. In COPD bronchial biopsies, Higham et al. [45] demonstrated lower levels of sub-mucosal eosinophilic inflammation are stable over time whereas higher levels of sub-mucosal eosinophilic inflammation are more variable. These results are similar to the stability of blood eosinophil counts which are used as a biomarker to guide inhaled corticosteroid (ICS) therapy. Pulmonary eosinophilic inflammation in COPD patients occurs in microenvironments enriched for other markers of type 2 inflammation including basophils, T-helper 2 lymphocytes and group 2 innate lymphoid cells [46]. These highly concentrated areas of type 2 inflammation may drive specific tissue remodelling. Indeed, eosinophil derived IL-13 has been shown to induce matrix metalloproteinase (MMP)-12 production from alveolar macrophages causing alveolar destruction in mice [47]. The authors of this study also demonstrated increased MMP-12 levels in COPD patients with eosinophilic inflammation and emphysema. Interestingly, COPD patients with an eosinophilic exacerbation had lower COPD Assessment Test scores compared to COPD patients with a bacterial exacerbation [48]. These data indicate specific pathophysiological impacts of eosinophilic inflammation which require further investigation.

Anti-viral immunity is reduced in COPD patients, increasing susceptibility to viral infections [49]. Whilst ICS dampen the anti-viral response from bronchial epithelial cells, COPD patients with a higher body mass index (BMI) experience more viral infections than those with a lower BMI (30 versus $27 \mathrm{~kg} \cdot \mathrm{m}^{-2}$ ) [50]. In a different study, increased abdominal adiposity in COPD patients was associated with a higher risk of hospitalisation or death [51]. Overall, fat mass appears to influence disease progression. One possible reason may be increased entry points for respiratory viruses. The expression of angiotensin converting enzyme (ACE)2, the receptor for severe acute respiratory syndrome (SARS), SARS-coronavirus (CoV)-2 and human CoV NL63 is increased in the bronchial epithelium of COPD patients with a higher BMI (29 versus $23 \mathrm{~kg} \cdot \mathrm{m}^{-2}$ ) [52]. However, data on other respiratory virus receptors is lacking. Alternatively, pulmonary adipose tissue may contribute to disease progression. ELLIOT et al. [53] demonstrated that peribronchial adipose tissue area was positively correlated with BMI and airway wall thickness in control and asthma patients. Furthermore, in fatal asthma cases, peribronchial adipose tissue area was positively correlated with the number of sub-epithelial eosinophils and neutrophils. Adipose tissue produces pro-inflammatory mediators which may cause further tissue inflammation and impact anti-microbial responses. Similar research should be conducted in COPD patients. 


\section{Maintenance pharmacotherapy}

From 2019, in the aftermath of the introduction of single inhaler triple therapy, there was generally more focus in the literature on the use of ICS. These debates were related to the right treatment for the right patient: patient characteristics and biomarkers that could predict response to treatment [54].

A post-hoc analysis from the single triple combination inhaler containing beclomethasone dipropionate (BDP), formoterol fumarate (FF) and glycopyrronium (G) (BDP/FF/G) programme (TRILOGY, TRINITY and TRIBUTE) showed that in the subgroup of patients with symptomatic COPD, severe or very severe airflow limitation, and a history of one moderate exacerbation, BDP/FF/G consistently reduced the risk on moderate-to-severe exacerbations by $22-23 \%$ in the three studies [55]. Similarly, HaLPIN et al. [56] investigated in a post-hoc analysis of the IMPACT (Informing the Pathway of COPD Treatment) trial that triple therapy (FF, umeclidinium (UMEC), vilanterol (VI)) was superior to both dual combination therapy (ICS-long-acting $\beta$-agonists (LABA) (FF/VI) and dual bronchodilation (UMEC/VI)) for preventing exacerbations and improving health status and forced expiratory volume in $1 \mathrm{~s}\left(\mathrm{FEV}_{1}\right)$, regardless of exacerbation history. These analyses therefore provide guidance on therapy for this group of symptomatic patients with only one exacerbation in the previous year who are often considered not at high risk of future exacerbations [57].

Halpin et al. [56] further reported that blood eosinophil counts helped to discriminate different treatment effects in patients at greater risk of exacerbations (i.e. patients with frequent moderate and severe exacerbations) but that eosinophil count was less useful in patients with a single moderate exacerbation in the preceding year. Analysis based on both prior exacerbation history and blood eosinophil counts showed no significant difference in exacerbation rates with triple therapy compared with dual therapy in patients with lower eosinophil counts irrespective of prior exacerbation history. Interestingly, the comparison of the dual combinations (ICS-LABA versus LABA-long-acting muscarinic antagonists (LAMA)) varied with previous exacerbation history and eosinophil count. Subjects with a single moderate exacerbation in the previous year had overall fewer exacerbations, with a less pronounced trend for ICS benefit at higher eosinophil counts compared to frequent exacerbators [56]. In line with this, a real-world matched historical cohort study that compared the effectiveness of triple therapy versus dual bronchodilation among patients with frequent exacerbations concluded that triple therapy was associated with a lower risk of oral corticosteroid prescriptions, and this was more pronounced with increased baseline eosinophil count, and previous exacerbation rate [58]. These results illustrate that combining previous exacerbation frequency and baseline eosinophil count helps in personalising inhaled treatment that reduces exacerbation risk.

Mathioudakis et al. [59] performed a post-hoc analysis of the ISOLDE trial, evaluating whether blood eosinophil change after ICS initiation could more accurately predict response to ICS than a single baseline eosinophil measurement; a decrease in blood eosinophils after ICS therapy was associated with a positive ICS treatment response, while an increase in eosinophils after ICS therapy revealed a patient group where ICS administration was associated with an increased risk of exacerbations and accelerated lung function decline. Having a prevalence of $20 \%$ within the study population, this cluster requires further evaluation. The authors speculate that the increased risk potentially relates to the immunosuppressive burden from ICS, in patients who do not gain benefit from them [59].

Jo et al. [60] investigated whether withdrawal compared to continuation of ICS was related to worse outcomes in terms of rehospitalisation or death or treatment for new exacerbations. Propensity score analyses were used in a retrospective cohort study of patients aged $\geqslant 65$ years who were hospitalised for COPD exacerbation using the Japanese Diagnosis Procedure Combination database from July 2010 to March 2016 [60]. There was a 30\% smaller risk of rehospitalisation or death and significantly less exacerbations in the group that discontinued ICS. This study suggests that a subgroup of patients might successfully discontinue ICS, but this study may have residual confounding as there is no information on the reason for discontinuation. Patients who stepped-down therapy might have been in a better general condition and ICS might have been considered not appropriate by the treating physician.

A ERS Task Force developed a guideline regarding ICS withdrawal in patients with COPD after appraising the evidence with the GRADE approach. In summary three recommendations were made: 1) conditional recommendation for the withdrawal of ICS in patients with COPD without a history of frequent exacerbations; 2) strong recommendation not to withdraw ICS in patients with blood eosinophil counts $\geqslant 300$ eosinophils $\mu \mathrm{L}^{-1}$; and 3) strong recommendation to treat with one or two long-acting bronchodilators if ICS are withdrawn [61].

An interesting study aimed to identify genetic loci that modify the effects of ICS on lung function in patients with COPD. This genome-wide association study was performed for genotype-by-ICS interaction effect on $\mathrm{FEV}_{1}$ decline in 802 subjects from the Lung Health Study. No single nucleotide polymorphisms (SNPs) met genome-wide significance $\left(\mathrm{p}<5 \times 10^{-8}\right)$. However, using a secondary defined criteria for 
discovery of $\mathrm{p}<5 \times 10^{-6}$, the authors identified an intergenic SNP on chromosome 7 (rs111720447, $\left.\mathrm{p}=4.81 \times 10^{-6}\right)$ that was replicated in the same direction $\left(\mathrm{p}=5.98 \times 10^{-5}\right)$ in an independent cohort $(\mathrm{n}=199)$ who were treated with either fluticasone alone or in combination with salmeterol versus placebo. These new findings may provide new insight into the potential biology of steroid responsiveness in COPD [62].

Several studies have recently evaluated the safety of ICS use. KENDZERSKA et al. [63] assessed pneumonia risk in a retrospective longitudinal population study of health administrative data from all insured individuals aged $\geqslant 66$ years in Canada between 2003 and 2014 who met a validated case definition of physician-diagnosed COPD (COPD cohort) and/or asthma (asthma cohort) and were new ICS users (as determined by a preceding 1-year ICS-free period). Over a median follow-up of 3.7 years, 27\% were hospitalised for asthma or COPD, and $23 \%$ were hospitalised for pneumonia. In an adjusted analysis, higher doses of ICS $(1000 \mu \mathrm{g}$ versus $500 \mu \mathrm{g})$ were not associated with improved effectiveness but with a higher risk for pneumonia hospitalisation (HR 1.06, 95\% CI 1.02-1.10). Budesonide and other ICS were associated with fewer pneumonia hospitalisations and fewer asthma or COPD hospitalisations than fluticasone. The authors rightfully mention that confounding due to fluticasone being more likely to be prescribed in higher dosages in individuals with more severe disease could not be excluded [63].

Another Canadian population-based linked administrative data between the years 1997 and 2007 from the province of British Columbia, evaluated the association between lung cancer risk and ICS use in COPD patients and concluded that sustained ICS exposure may be associated with a $30 \%$ reduced risk of lung cancer (HR 0.70, 95\% CI 0.61-0.80) [64]. Conversely, Suissa et al. [65] analysed the Quebec healthcare databases to identify COPD patients from 2000 to 2014 followed until 2015. The adjusted HR of lung cancer associated with ICS exposure was 1.01 (95\% CI 0.94-1.08), relative to no ICS use. SuISSA et al. [65] concluded that ICS use is not associated with a reduction in lung cancer incidence in patients with COPD and discussed that the reduction seen in the previous study may have been affected by immortal time bias.

The effectiveness of early introduction of LAMA for COPD was evaluated in patients who had completed the Tiotropium in Early-Stage COPD (Tie-COPD) trial. Participants continued or discontinued treatment according to their willingness and were followed for a maximum of 3 years. The follow-up study demonstrated an acute and persistent worsening after discontinuation of tiotropium, as indicated by $\mathrm{FEV}_{1}$, symptom score, quality-of-life score and acute exacerbation rate over a 1-year period [66]. These data argue for continuation of LAMA, even in early stage of COPD.

Prophylactic macrolide use was further evaluated in a post-hoc analysis of the previously published, double-blinded, placebo-controlled RCT MACRO in which azithromycin taken daily for 1 year reduced the risk of COPD exacerbations. The authors hypothesised that the subgroup of patients taking long-term azithromycin who experienced an index hospitalisation for an AECOPD would have a decreased risk of readmission when compared to the placebo arm. Patients receiving azithromycin did not have a significant increase in time to all-cause or respiratory-related readmission. Interestingly, increased adherence to prescribed drug, either azithromycin or placebo, was associated with increased time to readmission. The authors argue that this underlines the importance of adherence to therapy and introduces the healthy adherer effect, suggesting that adherence is an indicator of an overall healthy behaviour which may improve outcomes in RCTs [67].

Last but not least, SINGH et al. [68] reported positive results for a new kid on the block, CHF6001, an inhaled phosphodiesterase- 4 inhibitor, that in addition to beneficial clinical effects, successfully reduced sputum eosinophils in those COPD patients with higher eosinophil counts, supporting the evidence for targeting eosinophilic inflammation in COPD with (inhaled) phosphodiesterase-4 inhibitors.

\section{COPD exacerbation pharmacotherapy}

Two international research networks focusing on COPD exacerbations have emerged recently. The Collaboration In COPD ExaceRbatiOns (CICERO) ERS Clinical Research Collaboration (CRC) aims to develop an international prospective, longitudinal observational study to explore clinical and mechanistic characteristics of COPD exacerbations [69]. The DisEntangling Chronic Obstructive pulmonary Disease Exacerbations clinical trials NETwork (DECODE-NET) intends to launch multi-national RCTs to improve the management of COPD exacerbations, including precision medicine interventions, while improving the methodology of such trials [70].

COPD exacerbations are heterogeneous and only some are triggered by a bacterial infection, thus responding to the administration of antibiotics [4] and antibiotics are arguably overused in the absence of clinically validated, accurate, cost-effective therapeutic biomarkers [71]. PRINS et al. [72] reported an RCT evaluating whether C-reactive protein (CRP) can guide the administration of antibiotics for patients hospitalised with a COPD exacerbation. In the intervention group, antibiotics were only administered when CRP was $\geqslant 50 \mathrm{mg} \cdot \mathrm{L}^{-1}$ at presentation. That led to a modest (15\%) decrease in antibiotic 
administration without adversely impacting clinical outcomes such as the treatment failure rate, time to next exacerbation or length of stay. Identifying an appropriate CRP threshold for administering antibiotics is still needed, as another similar trial used a lower threshold [73]. While it appears that procalcitonin can safely, further decrease antibiotics administration, it is more expensive and not readily available to many clinical settings [71]. Other predictors of the need for antibiotics were evaluated in a post-hoc analysis from the TEXACOLD study. Interestingly, former smokers were three times more likely to experience treatment failure compared to current smokers [74]. Moreover, clinical characteristics of the participants, including the presence of sputum purulence, could not accurately predict the need for antibiotics.

Patient action plans for exacerbations were evaluated in a multinational RCT [75]. Within 1 year, this intervention reduced the duration of each exacerbation and the risk of having at least one respiratory-related hospitalisation. There was also a trend for fewer exacerbation days per patient per year. Overall, the effects were modest and, thus, caution is needed when implementing such interventions to avoid delaying the administration of more personalised treatments for specific exacerbations.

Extensive methodological systematic reviews identified significant methodological heterogeneity in the design of RCTs on the management of COPD exacerbations. First, there is very significant variability in the diagnostic criteria that are used for COPD exacerbations [76]. This is accompanied by a significant heterogeneity in the evaluated outcomes/end-points [32]. Only 63\% of all included trials reported on the rate of treatment success or failure, while only $42 \%$ reported on their duration [32]. These factors could limit the interpretability and comparability of the trial results. For this reason, an ERS Task Force was launched to produce a core outcome set for RCTs on the management of COPD exacerbations [77]. A core outcome set is an agreed minimum set of clinically important outcomes to be evaluated in all future relevant RCTs, with the aim to improve their usability and comparability.

\section{Physical activity, exercise and pulmonary rehabilitation}

Improving the physical fitness of individuals with COPD remains a key area of research, as evidenced by the wide spectrum of physical activity-related research presented at the 2019 ERS International Congress [14] and recent publications within the ERS journals [78].

Pulmonary rehabilitation, which incorporates measures to enhance physical fitness, delivers clear multifaceted benefits but many patients are not referred to, or do not attend these services. The multiple factors leading to these missed opportunities, and how to begin addressing them, can appear opaque to service providers. EARLY et al. [79] published eye-opening UK-based research reporting the referral and uptake barriers perceived by referrers, pulmonary rehabilitation providers and, crucially, patients (figure 2) [79]. Their mixed-methods study identified multiple potential service barriers relating to service design, referrer training, patient beliefs and data monitoring [79]. Importantly, alongside these barriers, the authors also identified important enablers. These insights will hopefully drive meaningful service and quality improvement, leading to more joined-up care delivery across the referrer, provider and patient boundaries.

Physical activity limitations among patients with COPD can be due to the inter-related factors of airflow obstruction, dynamic hyperinflation, impaired gas exchange, cardiac dysfunction and skeletal muscle dysfunction, manifesting as breathlessness and fatigue [22, 80]. Researchers continue to seek how best to target these different disease components to allow individually tailored therapies. Long-acting bronchodilators reduce hyperinflation and remain the mainstay of long-term COPD inhaler therapy. The OTIVATO trial investigated how, among patients with moderate-to-severe COPD and baseline hyperinflation, dual LAMA-LABA (tiotropium/olodaterol) bronchodilator versus monotherapy with a LAMA (tiotropium) impacted activity-related breathlessness [81]. Dual bronchodilator therapy both decreased activity-related breathlessness, measured using the modified Borg scale, after 3-min constant speed shuttle tests, and delivered substantial improvements in inspiratory capacity relative to monotherapy [81]. Although not formally tested as part of this study, relieving exertional breathlessness by optimising bronchodilator therapy may facilitate improved longer term physical activity and also enhance the benefits offered from the exercise training provided during pulmonary rehabilitation.

During physical activity, besides becoming increasingly hyperinflated, many patients with COPD also desaturate, contributing to activity limited dyspnoea. There was uncertainty as to whether correcting this during pulmonary rehabilitation exercise training would improve rehabilitation outcomes. Ausson et al. [82] investigated if $5 \mathrm{~L} \cdot \mathrm{min}^{-1}$ oxygen via nasal prongs improved endurance shuttle walk test time and Chronic Respiratory Disease Questionnaire total score among those who, although normoxic at baseline, desaturated to $<90 \%$ during exercise training. This large double blinded RCT found no difference in exercise improvements following training between the oxygen and medical air groups. While there remains debate regarding how different modalities of training may differentially benefit from supplementary oxygen [83, 84], by showing how both groups benefitted from pulmonary rehabilitation, this study 


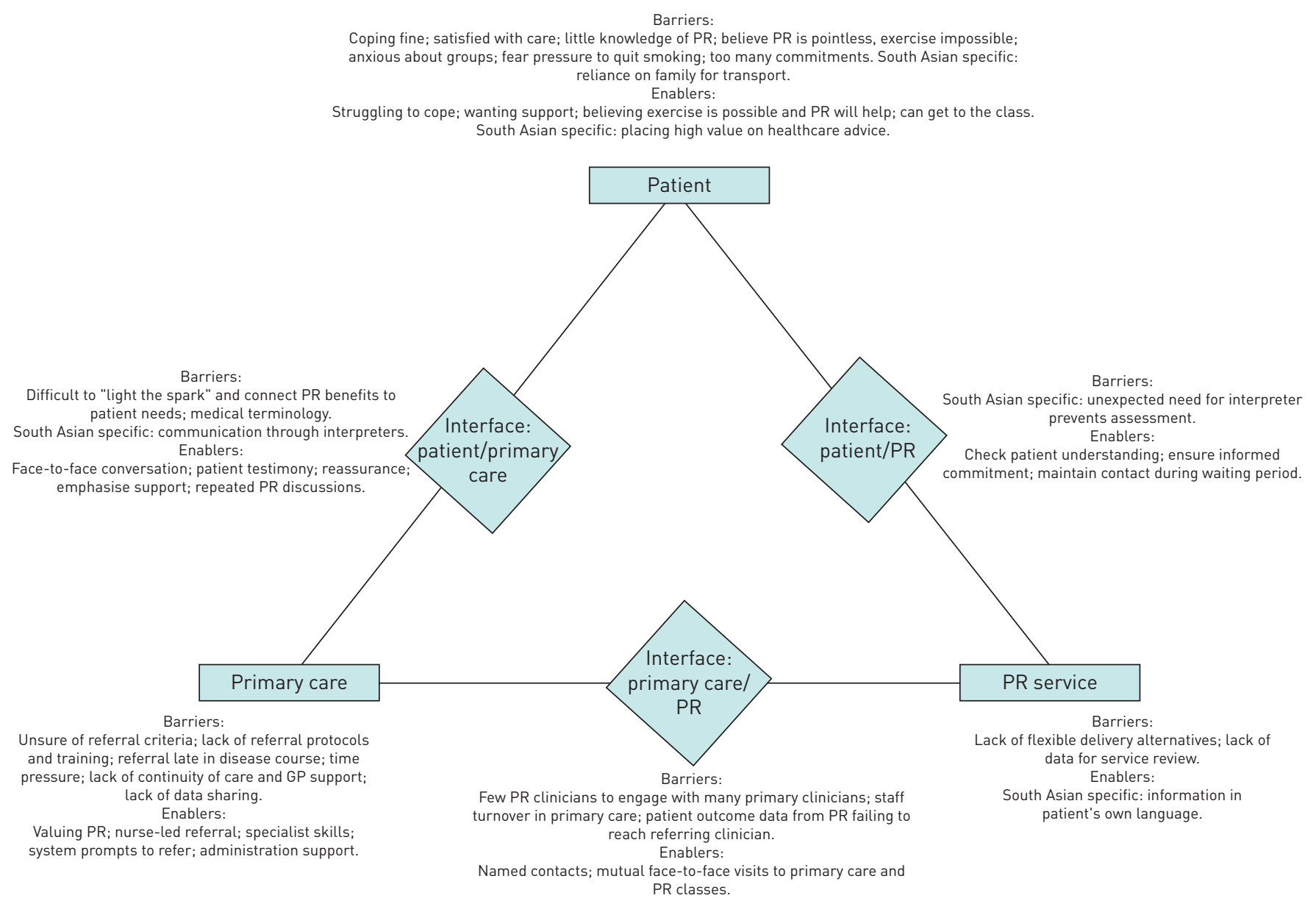

FIGURE 2 Barriers and enablers to pulmonary rehabilitation referral and uptake. PR: pulmonary rehabilitation; GP: general practitioner. Reproduced from [79] with permission.

reassures us that exercise training programmes can continue to be offered within venues without supplemental oxygen [82].

Although fatigue can limit exercise tolerance, achieving post-exercise contractile muscle fatigue may be important towards enhancing future exercise tolerance. However, many patients with COPD appear not to achieve contractile muscle fatigue during conventional pulmonary rehabilitation exercise training, perhaps because exercise is limited by other factors. CAmillo et al. [85] investigated how incorporating downhill walking training, rather than level walking, might help improve functional exercise tolerance. While the resulting differences in 6-min walk distance following rehabilitation were not significantly different ( $77 \pm 46 \mathrm{~m}$ versus $56 \pm 47 \mathrm{~m}$ in the downhill versus the level walking group $\mathrm{p}=0.45$ ), more patients from the downhill walking group exceeded the minimally importance difference following rehabilitation ( $94 \%$ versus $65 \%$, respectively; $\mathrm{p}=0.03$ ) [85]. The study also showed that this cheap and simple intervention was safe, suggesting that it is worthy of further research, especially given that this was a relatively small study.

COPD exacerbations can negatively impact health in numerous ways, including by inflicting a deterioration in physical performance and quality life. KJAERGAARD et al. [86] investigated if allocating patients to pulmonary rehabilitation within 2 weeks of discharge following hospital admission due to exacerbation, rather than after 2 months, improved physical activity outcomes. This RCT found that earlier pulmonary rehabilitation, while not altering mortality or hospital readmission rates, improved shuttle walk distance [86]. Early pulmonary rehabilitation following an acute exacerbation of COPD may therefore accelerate recovery in physical performance [86].

While pulmonary rehabilitation can deliver improvements in exercise tolerance, and many researchers focus upon how to maximise these improvements, these gains do not necessarily translate into longer term improvements in daily physical activity. Armstrong et al. [87] conducted a systematic review and 
meta-analysis of RCTs investigating how pedometers might be one tool which help improve physical activity among patients with COPD. Based on 19 RCTs which used pedometers either as a stand-alone intervention or in addition to pulmonary rehabilitation, they found that pedometers can improve daily physical activity [87]. They were more effective among patients with greater baseline physical activity [87]. Besides highlighting the patient subset who might most benefit from pedometers (the already more active patients), Armstrong et al. [87] highlighted the need to consider factors relating to cognition and mental health when trying to improve physical activity.

Adherence to interventions, such as pulmonary rehabilitation, can be negatively influenced by cognitive limitation and the psychosocial consequences of chronically symptomatic disease. In a secondary analysis of the PHYSACTO trial, LAvOIE et al. [88] incorporated a self-management behaviour modification (SMBM) programme into all four study arms (tiotropium-olodaterol and exercise training versus tiotropium-olodaterol only versus tiotropium only versus placebo) enrolling patients with moderate-to severe COPD. The SMBM programme aimed to increase participant motivation and confidence towards engaging in daily physical activity and the study found that all arms, even the arm with just SMBM, delivered clinically significant improvements in psychological and cognitive functioning [88]. Although not the primary focus of this study, this holistic approach may prove useful to helping deliver long-term behavioural changes which help individuals improve and sustain their physical activity.

\section{COPD and coronavirus disease 2019}

The interplay between coronavirus disease 2019 (COVID-19) and smoking or COPD has been extensively investigated. Studies have focused on the risk of contracting COVID-19, the clinical outcomes and appropriate treatment of patients with COPD during the pandemic. A preliminary meta-analysis of 11 case series in China and USA suggested that current smoking might be protective against contracting COVID-19 [89]. However, smoking status may have been under-reported in the included studies, which were mostly retrospective and based on routinely collected clinical data. In parallel, current smokers had higher prevalence of respiratory and cardiac diseases despite shielding measures to protect from contracting the virus [90-92]. From a mechanistic perspective, the expression of pulmonary ACE2, the entry receptor used by the novel coronavirus, is increased in COPD patients and cigarette smoking and obesity appear important risk factors, increasing the susceptibility to COVID-19 infection [52, 91-94]. For these reasons, absolute cessation of any tobacco product in any form is strongly recommended [95, 96].

There is strong evidence suggesting COPD is an important risk factor for adverse outcomes in patients with COVID-19 [91, 97]. In a retrospective study evaluating 1590 hospitalised patients with COVID-19, COPD was the strongest predictor for admission to the intensive care unit, invasive ventilation or death [98]. COPD was also a risk factor for hospitalisation and adverse outcomes in a series of 90000 unselected patients diagnosed with COVID-19 in Mexico [99]. Numerous extensive and well-conducted cohort studies globally confirmed that COPD is an independent risk factor for hospitalisation, intensive care admission, ventilation and death [91]. Therefore, there is a need for shielding and early vaccination, once effective vaccines become available, for these susceptible patients.

Finally, the role of ICS in COPD therapeutics in the COVID-19 era has been discussed. Mechanistically, corticosteroids suppress the production of anti-viral interferon type 1 and 3 from the epithelial cells, which is associated with increased viral replication and excess mucin production [49, 91]. However, in selected patients with COPD, ICS use is associated with a decreased rate of exacerbations and a better quality of life [2]. More importantly, ICS can prevent hospital admissions (for COPD exacerbations) [2], and this is a crucial benefit, especially during the unfolding of the COVID-19 pandemic. In parallel, a recent systematic review revealed a paucity of clinical data on the impact of ICS on the outcomes of SARS, SARS-CoV-2 or Middle East Respiratory Syndrome [100]. Therefore, in view of the well-established clinical benefits of ICS, there is consensus that ICS use should continue when indicated, in line with GOLD recommendations, in anticipation of further, direct clinical evidence [100].

Conflict of interest: A.G. Mathioudakis has nothing to disclose. L.E.G.W. Vanfleteren reports grants and personal fees from AstraZeneca, and personal fees from Novartis, GSK, Chiesi, Menarini, Pulmonx, Resmed, Boehringer, Verona Pharma and AGA Linde outside the submitted work. L. Lahousse reports Society awards sponsored by AstraZeneca and Chiesi; and has provided expert consultation for Boehringer Ingelheim GmbH and Novartis, outside the submitted work. A. Higham reports personal fees from Chiesi Pharmaceuticals, outside the submitted work. J.P. Allinson reports personal fees from Pulmonx, and non-financial support from GSK and Boehringer Ingelheim, outside the submitted work. C. Gotera has nothing to disclose. D. Visca has nothing to disclose. D. Singh reports grants and personal fees from AstraZeneca, Boehringer Ingelheim, Chiesi, GlaxoSmithKline, Glenmark, Menarini, Mundipharma, Novartis, Pfizer, Pulmatrix, Therevance and Verona, and personal fees from Cipla, Genentech and Peptinnovate, outside the submitted work. A. Spanevello has nothing to disclose.

Support statement: A.G. Mathioudakis, A. Higham and D. Singh were supported by the NIHR Manchester Biomedical Research Centre (BRC). 


\section{References}

1 GBD 2016 Causes of Death Collaborators. Global, regional, and national age-sex specific mortality for 264 causes of death, 1980-2016: a systematic analysis for the Global Burden of Disease Study 2016. Lancet 2017; 390: 1151-1210.

2 Singh D, Agusti A, Anzueto A, et al. Global Strategy for the Diagnosis, Management, and Prevention of Chronic Obstructive Lung Disease: the GOLD science committee report 2019. Eur Respir J 2019; 53: 1900164.

3 Agusti A, Bel E, Thomas M, et al. Treatable traits: toward precision medicine of chronic airway diseases. Eur Respir J 2016; 47: 410-419.

4 Mathioudakis AG, Janssens W, Sivapalan P, et al. Acute exacerbations of chronic obstructive pulmonary disease: in search of diagnostic biomarkers and treatable traits. Thorax 2020; 75: 520-527.

5 Schleich F, Bikov A, Mathioudakis AG, et al. Research highlights from the 2018 European Respiratory Society International Congress: airway disease. ERJ Open Res 2019; 5: 00225-2018.

6 Lahousse L, Bahmer T, Cuevas-Ocaña S, et al. ERS International Congress, Madrid, 2019: highlights from the Airway Diseases, Asthma and COPD Assembly. ERJ Open Res 2020; 6: 00341-2019.

7 Crespo A, Baillieul S, Marhuenda E, et al. ERS International Congress, Madrid, 2019: highlights from the Sleep and Clinical Physiology Assembly. ERJ Open Res 2020; 6: 00373-2019.

8 Vanfleteren L, Blervaque L, Franssen FME, et al. ERS International Congress, Madrid, 2019: highlights from the General Pneumology Assembly. ERJ Open Res 2020; 6: 00323-2019.

9 Satici C, Lopez-Padilla D, Schreiber A, et al. ERS International Congress, Madrid, 2019: highlights from the Respiratory Intensive Care Assembly. ERJ Open Res 2020; 6: 00331-2019.

10 Calarasu C, Chichirelo-Konstantynovych KD, Frent S. ERS International Congress, Madrid, 2019: highlights from the Respiratory Infections Assembly. ERJ Open Res 2020; 6: 00316-2019.

11 Costantini A, Bostantzoglou C, Blum TG. ERS International Congress, Madrid, 2019: highlights from the Thoracic Oncology Assembly. ERJ Open Res 2020; 6: 00131-2020.

12 Nenna R, Slaats M, Ersu R, et al. ERS International Congress, Madrid, 2019: highlights from the Paediatric Assembly. ERJ Open Res 2020; 6: 00063-2020.

13 d'Hooghe J, Alvarez Martinez H, Pietersen PI, et al. ERS International Congress, Madrid, 2019: highlights from the Clinical Techniques, Imaging and Endoscopy Assembly. ERJ Open Res 2020; 6: 00116-2020.

14 Oliveira A, Rutter M, Quijano-Campos JC, et al. ERS International Congress, Madrid, 2019: highlights from the Allied Respiratory Professionals' Assembly. ERJ Open Res 2020; 6: 00034-2020.

15 Blanco I, Diego I, Bueno P, et al. Geographic distribution of COPD prevalence in the world displayed by Geographic Information System maps. Eur Respir J 2019; 54: 1900610.

16 Halpin DMG, Celli BR, Criner GJ, et al. It is time for the world to take COPD seriously: a statement from the GOLD board of directors. Eur Respir J 2019; 54: 1900914.

17 Soriano JB. The evolution of COPD species; or, something is changing for good in COPD. Eur Respir J 2019; 53: 1900762 .

18 Doiron D, de Hoogh K, Probst-Hensch N, et al. Air pollution, lung function and COPD: results from the population-based UK Biobank study. Eur Respir J 2019; 54: 1802140.

19 De Matteis S, Jarvis D, Darnton A, et al. The occupations at increased risk of COPD: analysis of lifetime job-histories in the population-based UK Biobank Cohort. Eur Respir J 2019; 54: 1900186.

20 Brakema EA, Tabyshova A, Kasteleyn MJ, et al. High COPD prevalence at high altitude: does household air pollution play a role? Eur Respir J 2019; 53: 1801193.

21 Gut-Gobert C, Cavaillès A, Dixmier A, et al. Women and COPD: do we need more evidence? Eur Respir Rev 2019; 28: 180055.

22 Butler SJ, Li LSK, Ellerton L, et al. Prevalence of comorbidities and impact on pulmonary rehabilitation outcomes. ERJ Open Res 2019; 5: 00264-2019.

23 Boehm A, Pizzini A, Sonnweber T, et al. Assessing global COPD awareness with Google Trends. Eur Respir J 2019; 53: 1900351.

24 Soriano JB, Ancochea J, Celli BR. The most beautiful COPD chart in the world: all together to end COPD!. Eur Respir J 2019; 54: 1902047.

25 Goedemans L, Bax JJ, Delgado V. COPD and acute myocardial infarction. Eur Respir Rev 2020; 29 : 190139.

26 Wijnant SRA, De Roos E, Kavousi M, et al. Trajectory and mortality of preserved ratio impaired spirometry: the Rotterdam Study. Eur Respir J 2020; 55: 1901217.

27 Waschki B, Alter P, Zeller T, et al. High-sensitivity troponin I and all-cause mortality in patients with stable COPD: an analysis of the COSYCONET study. Eur Respir J 2020; 55: 1901314.

28 Keene SJ, Adab P, de Vries F, et al. The stability of the ADO score among UK COPD patients from The Health Improvement Network. ERJ Open Res 2020; 6: 00196-2019.

29 Stockley RA, Halpin DMG, Celli BR, et al. chronic obstructive pulmonary disease biomarkers and their interpretation. Am J Respir Crit Care Med 2019; 199: 1195-1204.

30 Martin RJ, Bel EH, Pavord ID, et al. Defining severe obstructive lung disease in the biologic era: an endotype-based approach. Eur Respir J 2019; 54: 1900108.

31 Benz E, Trajanoska K, Lahousse L, et al. Sarcopenia in COPD: a systematic review and meta-analysis. Eur Respir Rev 2019; 28: 190049.

32 Mathioudakis AG, Moberg M, Janner J, et al. Outcomes reported on the management of COPD exacerbations: a systematic survey of randomised controlled trials. ERJ Open Res 2019; 5: 00072-2019.

33 Koo HK, Vasilescu DM, Booth S, et al. Small airways disease in mild and moderate chronic obstructive pulmonary disease: a cross-sectional study. Lancet Respir Med 2018; 6: 591-602.

34 Capron T, Bourdin A, Perez T, et al. COPD beyond proximal bronchial obstruction: phenotyping and related tools at the bedside. Eur Respir Rev 2019; 28: 190010.

35 Brightling C, Greening N. Airway inflammation in COPD: progress to precision medicine. Eur Respir J 2019; 54: 1900651.

36 Hogg JC, Chu F, Utokaparch S, et al. The nature of small-airway obstruction in chronic obstructive pulmonary disease. N Engl J Med 2004; 350: 2645-2653. 
Wooding DJ, Ryu MH, Li H, et al. Acute air pollution exposure alters neutrophils in never-smokers and at-risk humans. Eur Respir J 2020; 55: 1901495.

Knabe L, Petit A, Vernisse C, et al. CCSP counterbalances airway epithelial-driven neutrophilic chemotaxis. Eur Respir J 2019; 54: 1802408.

Starkey MR, Plank MW, Casolari P, et al. IL-22 and its receptors are increased in human and experimental COPD and contribute to pathogenesis. Eur Respir J 2019; 54: 1800174.

Singh D. Chronic obstructive pulmonary disease, neutrophils and bacterial infection: a complex web involving IL-17 and IL-22 unravels. EBioMedicine 2015; 2: 1580-1581.

Zuo WL, Yang J, Strulovici-Barel Y, et al. Exaggerated BMP4 signalling alters human airway basal progenitor cell differentiation to cigarette smoking-related phenotypes. Eur Respir J 2019; 53: 1702553.

Lin VY, Kaza N, Birket SE, et al. Excess mucus viscosity and airway dehydration impact COPD airway clearance. Eur Respir J 2020; 55: 1900419.

Dupin I, Thumerel M, Maurat E, et al. Fibrocyte accumulation in the airway walls of COPD patients. Eur Respir J 2019; 54: 1802173.

Papakonstantinou E, Bonovolias I, Roth M, et al. Serum levels of hyaluronic acid are associated with COPD severity and predict survival. Eur Respir J 2019; 53: 1801183.

Higham A, Leow-Dyke S, Jackson N, et al. Stability of Eosinophilic Inflammation in COPD Bronchial Biopsies. Eur Respir J 2020; 56: 2000622.

Jogdand P, Siddhuraj P, Mori M, et al. Eosinophils, basophils and type 2 immune microenvironments in COPD affected lung tissue. Eur Respir J 2020; 55: 1900110.

Doyle AD, Mukherjee M, LeSuer WE, et al. Eosinophil-derived IL-13 promotes emphysema. Eur Respir J 2019; 53: 1801291.

Keir HR, Dicker A, Lonergan M, et al. Clinical endotypes of exacerbation are associated with differences in microbial composition and diversity in COPD. Eur Respir J 2020; 56: 2000391.

Singanayagam A, Glanville N, Girkin JL, et al. Corticosteroid suppression of antiviral immunity increases bacterial loads and mucus production in COPD exacerbations. Nat Commun 2018; 9: 2229.

Stolz D, Papakonstantinou E, Grize L, et al. Time-course of upper respiratory tract viral infection and COPD exacerbation. Eur Respir J 2019; 54: 1900407.

Li J, Zhu L, Wei Y, et al. Association between adiposity measures and COPD risk in Chinese adults. Eur Respir J 2020; 55: 1901899.

Higham A, Singh D. Increased ACE2 expression in bronchial epithelium of COPD patients who are overweight. Obesity (Silver Spring) 2020; 28: 1586-1589.

Elliot JG, Donovan GM, Wang KCW, et al. Fatty airways: implications for obstructive disease. Eur Respir J 2019; 54: 1900857.

Agusti A, Fabbri LM, Singh D, et al. Inhaled corticosteroids in COPD: friend or foe? Eur Respir J 2018; 52 1801219.

Singh D, Fabbri LM, Corradi M, et al. Extrafine triple therapy in patients with symptomatic COPD and history of one moderate exacerbation. Eur Respir J 2019; 53: 1900235.

Halpin DMG, Dransfield MT, Han MK, et al. The effect of exacerbation history on outcomes in the IMPACT trial. Eur Respir J 2020; 55: 1901921.

Agusti A, Alcazar B, Cosio B, et al. Time for a change: anticipating the diagnosis and treatment of COPD. Eur Respir J 2020; 56: 2002104.

Voorham J, Corradi M, Papi A, et al. Comparative effectiveness of triple therapy versus dual bronchodilation in COPD. ERJ Open Res 2019; 5: 00106-2019.

Mathioudakis AG, Bikov A, Foden P, et al. Change in blood eosinophils following treatment with inhaled corticosteroids may predict long-term clinical response in COPD. Eur Respir J 2020; 55: 1902119.

Jo $\mathrm{T}$, Yasunaga $\mathrm{H}$, Yamauchi $\mathrm{Y}$, et al. Inhaled corticosteroid withdrawal may improve outcomes in elderly patients with COPD exacerbation: a nationwide database study. ERJ Open Res 2020; 6: 00246-2019.

Chalmers JD, Laska IF, Franssen FME, et al. Withdrawal of inhaled corticosteroids in COPD: a European Respiratory Society guideline. Eur Respir J 2020; 55: 2000351.

Obeidat M, Faiz A, Li X, et al. The pharmacogenomics of inhaled corticosteroids and lung function decline in COPD. Eur Respir J 2019; 54: 1900521.

Kendzerska T, Aaron SD, To T, et al. The Effect of Type and Dosage of Newly Prescribed Inhaled Corticosteroids on Obstructive Lung Disease and Pneumonia Hospitalisations in Older Individuals with Asthma, Chronic Obstructive Pulmonary Disease (COPD) or Both: A Retrospective Study of Health Administrative Data. Eur Respir J 2020; in press [http://doi.org/10.1183/13993003.02585-2020].

Raymakers AJN, Sadatsafavi M, Sin DD, et al. Inhaled corticosteroids and the risk of lung cancer in COPD: a population-based cohort study. Eur Respir J 2019; 53: 1801257.

Suissa S, Dell'Aniello S, Gonzalez AV, et al. Inhaled corticosteroid use and the incidence of lung cancer in COPD. Eur Respir J 2020; 55: 1901720.

Li C, Zhou Y, Liu S, et al. Tiotropium discontinuation in patients with early-stage COPD: a prospective observational cohort study. ERJ Open Res 2019; 5: 00175-2018.

Krishnan JK, Voelker H, Connett JE, et al. Effect of daily azithromycin therapy and adherence on readmission risk in COPD. Eur Respir J 2019; 53: 1801377.

Singh D, Watz H, Beeh KM, et al. COPD sputum eosinophils: relationship to blood eosinophils and the effect of inhaled PDE4 inhibition. Eur Respir J 2020; 56: 2000237.

Janssens W, Bafadhel M, The CICERO (Collaboration In COPD ExaceRbatiOns) Clinical Research Collaboration. Eur Respir J 2020; 55: 2000079.

Mathioudakis AG, Sivapalan P, Papi A, et al. The DisEntangling Chronic Obstructive pulmonary Disease Exacerbations clinical trials NETwork: DECODE-NET. Rationale and vision. Eur Respir J 2020; 56: 2000627.

Mathioudakis AG, Chatzimavridou-Grigoriadou V, Corlateanu A, et al. Procalcitonin to guide antibiotic administration in COPD exacerbations: a meta-analysis. Eur Respir Rev 2017; 26: 160073.

Prins HJ, Duijkers R, van der Valk P, et al. CRP-guided antibiotic treatment in acute exacerbations of COPD in hospital admissions. Eur Respir J 2019; 53: 1802014. 
Butler CC, Gillespie D, White P, et al. C-reactive protein testing to guide antibiotic prescribing for COPD exacerbations. N Engl J Med 2019; 381: 111-120.

van Velzen P, Ter Riet G, Brinkman P, et al. Doxycycline for exacerbations of chronic obstructive pulmonary disease in outpatients: who benefits? ERJ Open Res 2020; 6: 00099-2020.

Lenferink A, van der Palen J, van der Valk PDLPM, et al. Exacerbation action plans for patients with COPD and comorbidities: a randomised controlled trial. Eur Respir J 2019; 54: 1802134.

Mathioudakis AG, Janner J, Moberg M, et al. A systematic evaluation of the diagnostic criteria for COPD and exacerbations used in randomised controlled trials on the management of COPD exacerbations. ERJ Open Res 2019; 5: 00136-2019.

Mathioudakis AG, Abroug F, Agusti A, et al. Core Outcome Set for the management of Acute Exacerbations of Chronic Obstructive Pulmonary Disease. The COS-AECOPD ERS Task Force study protocol. ERJ Open Res 2020; 6: 00193-2020.

Kong CW, Wilkinson TMA. Predicting and preventing hospital readmission for exacerbations of COPD. ERJ Open Res 2020; 6: 00325-2019.

Early F, Wilson PM, Deaton C, et al. Pulmonary rehabilitation referral and uptake from primary care for people living with COPD: a mixed-methods study. ERJ Open Res 2020; 6: 00219-2019.

impaired aerobic function. Eur Respir J 2019; 53: 1802386

Maltais F, Aumann JL, Kirsten AM, et al. Dual bronchodilation with tiotropium/olodaterol further reduces activity-related breathlessness versus tiotropium alone in COPD. Eur Respir J 2019; 53: 1802049.

Alison JA, McKeough ZJ, Leung RWM, et al. Oxygen compared to air during exercise training in COPD with exercise-induced desaturation. Eur Respir J 2019; 53: 1802429.

Langer D, Gosselink R. Why does oxygen supplementation during exercise training in COPD patients with exercise-induced desaturation not consistently improve exercise capacity? Eur Respir J 2019; 54: 1901586.

Alison JA, McKeough ZJ, Leung RWM, et al. Exercise training in COPD with exercise-induced desaturation does improve exercise capacity, irrespective of whether supplemental oxygen or air is provided during training. Eur Respir J 2019; 54: 1901725.

Camillo CA, Osadnik CR, Burtin C, et al. Effects of downhill walking in pulmonary rehabilitation for patients with COPD: a randomised controlled trial. Eur Respir J 2020; 56: 2000639.

Kjaergaard JL, Juhl CB, Lange P, et al. Early pulmonary rehabilitation after acute exacerbation of COPD: a randomised controlled trial. ERJ Open Res 2020; 6: 00173-2019.

Armstrong M, Winnard A, Chynkiamis N, et al. Use of pedometers as a tool to promote daily physical activity levels in patients with COPD: a systematic review and meta-analysis. Eur Respir Rev 2019; 28: 190039.

Lavoie KL, Sedeno M, Hamilton A, et al. Behavioural interventions targeting physical activity improve psychocognitive outcomes in COPD. ERJ Open Res 2019; 5: 00013-2019.

Farsalinos K, Angelopoulou A, Alexandris N, et al. COVID-19 and the nicotinic cholinergic system. Eur Respir J 2020; 56: 2001589.

Leung JM, Yang CX, Sin DD. Current smoking is not associated with COVID-19. Eur Respir J 2020; 55: 2001340.

Higham A, Mathioudakis AG, Vestbo J, et al. COVID-19 and COPD: a narrative review of the basic science and clinical outcomes. Eur Respir Rev 2020; 29: 200199.

Leung JM, Sin DD. Smoking, ACE-2 and COVID-19: ongoing controversies. Eur Respir J 2020; 56: 2001759

Jacobs M, Van Eeckhoutte HP, Wijnant SRA, et al. Increased expression of ACE2, the SARS-CoV-2 entry receptor, in alveolar and bronchial epithelium of smokers and COPD subjects. Eur Respir J 2020; 56: 2002378.

Leung JM, Yang CX, Sin DD. COVID-19 and nicotine as a mediator of ACE-2. Eur Respir J 2020; 55: 2001261. infection? Eur Respir J 2020; 56: 2001645.

Boutou AK, Pitsiou G, Kontakiotis T, et al. Nicotine treatment and smoking cessation in the era of COVID-19 pandemic: an interesting alliance. ERJ Open Res 2020; 6: 00306-2020.

Leung JM, Niikura M, Yang CWT, et al. COVID-19 and COPD. Eur Respir J 2020; 56: 2002108.

Guan WJ, Liang WH, Zhao Y, et al. Comorbidity and its impact on 1590 patients with COVID-19 in China: a nationwide analysis. Eur Respir J 2020; 55: 2000547.

Giannouchos TV, Sussman RA, Mier JM, et al. Characteristics and risk factors for COVID-19 diagnosis an adverse outcomes in Mexico: an analysis of 89,756 laboratory-confirmed COVID-19 cases. Eur Respir J 2020; in press [http://doi.org/10.1183/13993003.02144-2020].

Halpin DMG, Singh D, Hadfield RM. Inhaled corticosteroids and COVID-19: a systematic review and clinical perspective. Eur Respir J 2020; 55: 2001009. 